\title{
Sanity check for $N N$ bound states in lattice QCD with Lüscher's finite volume formula - Disclosing Symptoms of Fake Plateaux -
}

\author{
Sinya Aoki ${ }^{1,2, \star, \star \star}$, Takumi Doi ${ }^{3}$, and Takumi Iritani ${ }^{3}$ \\ ${ }^{1}$ Center for Gravitational Physics, Yukawa Institute for Theoretical Physics, Kyoto University, Kitashirakawa \\ Oiwakecho, Sakyo-ku, Kyoto 606-8502, Japan \\ ${ }^{2}$ Center for Computational Sciences, University of Tsukuba, Tsukuba 305-8577, Japan \\ ${ }^{3}$ Theoretical Research Division, Nishina Center, RIKEN, Wako 351-0198, Japan
}

\begin{abstract}
The sanity check is to rule out certain classes of obviously false results, not to catch every possible error. After reviewing such a sanity check for $N N$ bound states with the Lüscher's finite volume formula [1-3], we give further evidences for the operator dependence of plateaux, a symptom of the fake plateau problem, against the claim [4]. We then present our critical comments on [5] by NPLQCD: (i) Operator dependences of plateaux in NPL2013 [6, 7] exist with the $P$ value of 4-5\%. (ii) The volume independence of plateaux in NPL2013 does not prove their correctness. (iii) Effective range expansions (EREs) in NPL2013 violate the physical pole condition. (iv) Their comment is partly based on new data and analysis different from the original ones. (v) Their new ERE does not satisfy the Lüscher's finite volume formula.
\end{abstract}

\section{Introduction}

In the previous publications[1-3], we pointed out that the binding energy of the two nucleon $(N N)$ system at heavy pion masses extracted from the temporal correlation function at $t \simeq 1 \mathrm{fm}$ in lattice QCD is unreliable due to contaminations of excited scattering states. Tab. 1 summarizes our sanity check to recent $N N$ data, all of which employ the plateau fitting at $t \simeq 1 \mathrm{fm}$. The sanity check is performed to rule out certain classes of obviously false results, but the correctness is not established even if the data pass the test. A fact that none in the table passes the test brings a serious doubt on an existence of $N N$ bound states in ${ }^{1} S_{0}$ or ${ }^{3} S_{1}$ claimed on the basis of these data. Therefore, more sophisticated method than the simple plateau fitting, such as the variational method [9], is mandatory for the reliable study of $N N$ systems.

It seems, however, that our concerns mentioned in Refs. [1-3] are misunderstood by authors in Refs. $[4,5]$, as they tried to show that their data pass the sanity check. Therefore we will stress

\footnotetext{
${ }^{\star}$ Speaker, e-mail: saoki@yukawa.kyoto-u.ac.jp

${ }^{\star}{ }^{\star}$ Supported in part by the Grant-in-Aid of the Japanese Ministry of Education, Sciences and Technology, Sports and Culture (MEXT) for Scientific Research (No. JP16H03978), by a priority issue (Elucidation of the fundamental laws and evolution of the universe) to be tackled by using Post "K" Computer, and by Joint Institute for Computational Fundamental Science (JICFuS).
} 
Table 1. A summary of our assessment (Source independence, (i) consistency (ii) regularity (iii) physical pole). Here "No" means that the check fails while $\dagger$ implies no study on the item. See Ref. [3] for more details.

\begin{tabular}{|c||c|c|c|c|c|c|c|c|}
\hline \multicolumn{1}{|c||}{} & \multicolumn{4}{c|}{$N N\left({ }^{1} S_{0}\right)$} & \multicolumn{4}{c|}{$N N\left({ }^{3} S_{1}\right)$} \\
\hline Data & Source & \multicolumn{2}{c|}{ Sanity check } & \multicolumn{2}{c|}{ Source } & \multicolumn{2}{c|}{ Sanity check } \\
& independence & (i) & (ii) & (iii) & independence & (i) & (ii) & (iii) \\
\hline \hline YKU2011 [10] & $\dagger$ & No & No & & $\dagger$ & No & No & \\
YIKU2012 [11] & No & $\dagger$ & No & & No & $\dagger$ & No & \\
YIKU2015 [12] & $\dagger$ & $\dagger$ & No & & $\dagger$ & $\dagger$ & No & No \\
NPL2012 [13] & $\dagger$ & $\dagger$ & No & & $\dagger$ & $\dagger$ & & \\
NPL2013 [6, 7] & No & & & No & No & & & No \\
NPL2015 [14] & $\dagger$ & No & & No & $\dagger$ & No & & No \\
CalLat2017 [15] & No & $?$ & & No & No & $?$ & & No \\
\hline
\end{tabular}

again the serious problem of the plateau fitting and then give critical comments on Refs. $[4,5]$ in the remaining of this report.

\section{Fake plateau problem}
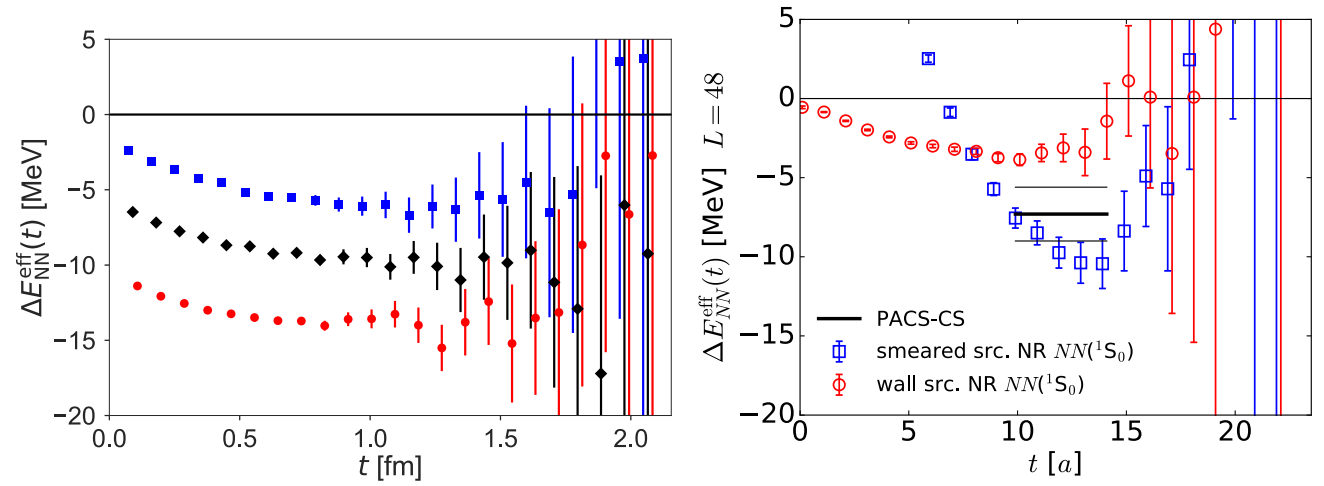

Figure 1. (Left) $\Delta E_{N N}^{\mathrm{eff}}(t)$ with fluctuations and errors as a function of $t$. (Right) $\Delta E_{N N}^{\mathrm{eff}}(t)$ from the wall source (red circles) and the smeared source (blue squares).

Plateaux of the $N N$ effective energy shift at early $t \simeq 1 \mathrm{fm}$ cannot be trusted in principle, since the fake plateaux can easily appear due to contaminations from elastic excited states. To demonstrate this, we consider the mock-up data for the effective energy shift, described by

$$
\Delta E_{N N}^{\mathrm{eff}}(t)=\frac{1}{a} \log \left(\frac{R(t+a)}{R(t)}\right), \quad R(t)=e^{-\Delta E_{N N} t}\left(1+b_{1} e^{-\delta E_{\mathrm{el} .} t}+c_{0} e^{-\delta E_{\text {inel. } t} t} .\right.
$$

with the correct energy shift $\Delta E_{N N}$, where we take $\delta E_{\text {el. }}=50 \mathrm{MeV}$, which is the typical lowest elastic excitation energy at $L \simeq 4 \mathrm{fm}$ (and $m_{N} \simeq 2 \mathrm{GeV}$ ), and $\delta E_{\text {inel. }}=500 \mathrm{MeV}$, which is the mass of the heavy pion. In this situation, we naively expect that the ground state saturation requires $t \geq 1 / \delta E_{\text {el. }} \simeq 4$ $\mathrm{fm}$, which however is too large to have good signals for $N N$ systems. The strategy used in the previous studies is tuning the source operators to satisfy $b_{1} \simeq 0$, so as to achieve the ground state saturation at 
much smaller $t$ before noises dominates ('"the golden window"). In practice, the source operator is tuned to have a plateau in $\Delta E_{N N}^{\mathrm{eff}}(t)$ at muh smaller $t$.

Fig. 1 (Left) shows three examples of $\Delta E_{N N}^{\mathrm{eff}}(t)$ with $b_{1}=0$ (an optimally tuned operator) and $b_{1}= \pm 0.1$ keeping $c_{0}=0.01$, where random fluctuations are assigned to $R(t)$ whose magnitude increases exponentially in $t$. Indeed the fake plateaux appear at $t \simeq 1 \mathrm{fm}$. If no legend of data is given as in the figure, we can not tell which are fake from the $t$ dependence.

This example clearly demonstrates that the strategy mentioned above does NOT work at all: Even if the plateau appears in $\Delta E_{N N}^{\mathrm{eff}}(t)$, we cannot tell whether it is fake or not. Hereafter we will use the words, "the fake plateau problem", to remind readers of this fundamental problem, which is the serious issue for the current $N N$ data and nothing more is needed to doubt the validity of data in Refs. [6, 7, 10-15]. Authors of these references should take full responsibility to prove correctness of their data, without relying on the "early plateau" strategy mentioned above.

\section{Operator dependence}

As manifestations of the "fake plateau problem", there exist several symptoms, one of which is the operator dependence of the plateau. Fig. 1 (Right) gives $\Delta E_{N N}^{\text {eff }}(t)$ from wall and smeared sources, which shows plateaux at different values[1], so that at least one of them must be fake.

Authors of Ref. [4] claimed that the plateau in $\Delta E_{N N}^{\mathrm{eff}}(t)$ from the wall source is fake, caused by a cancellation between the $N N$ effective energy and the nucleon effective mass, both of which seem to reach plateau slower than $\Delta E_{N N}^{\text {eff }}(t)$. This argument, however, does not properly address the issue in Ref. [1] as discussed below.

There is no reason for the smeared source works better than the wall source for the $N N$ system, since the smeared source is known to suppress inelastic contributions to nucleon mass but to couple strongly to elastic scattering states with non-zero momenta. Therefore, even if the plateau from the wall source were fake, it would NOT mean that the plateau from the smeared source is correct. In fact, as shown in Fig. 2 (Left), where the effective energy of $\Xi \Xi\left({ }^{1} S_{0}\right)$ is plotted for several different sink operators, ${ }^{1}$ the smeared source produces several different plateaux, (almost) all of which should be fake. This clearly shows that the plateau method does not have a predictive power at all.

In this conference [16], Berkowitz and his collaborators pointed out a possibility that inelastic excited state contributions in the nucleon effective mass may be largely cancelled by those in the $N N$ effective energy, so that the plateau may appear at earlier $t$ in $\Delta E_{N N}^{\text {eff }}(t)$ as long as contaminations from elastic excited states in the $N N$ effective energy are negligible. This interesting possibility, however, should be verified by more reliable methods such as variational method [9], since the simple plateau method cannot confirm it.

In Ref. [4], authors also claimed that the wall source and the smeared source agree at larger $t$ within large errors, as for data at $t \geq 16$ in Fig. 1 (Left). In this case, however, one has to fit data at $t \geq 16$, leading to a different central value with much larger errors than before (solid black lines).

It is useless to speculate a possible scenario for results from the plateau method to be correct without clear evidences. Instead one has to show that they are indeed correct without relying on the plateau method. The issue is the "fake plateau problem", not the wall source against the smeared source unlike the argument in Ref. [4]. i

\footnotetext{
${ }^{1} \Xi \Xi$ has smaller statistical errors than $N N$. See Appendix A in Ref. [1] for details.
} 

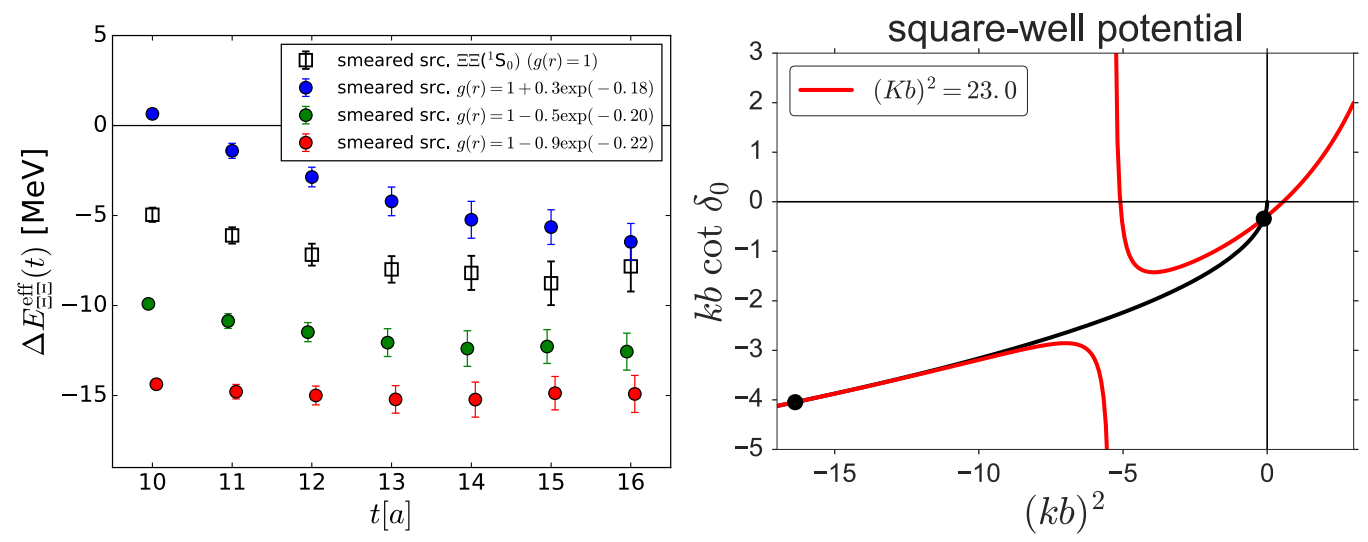

Figure 2. (Left) The effective energy shift $\Delta E_{\Xi \Xi}^{\text {eff }}(t)$ from the smeared source with different sink operators. (Right) An example of physically allowed EREs compatible with two bound states in the case of the square-well potential.

\section{Comment on Ref. [5]}

Authors of Ref. [5] claimed that the assessment in Ref. [3] to NPL2013 [6, 7] is inadequate and NPL2013 has passed all tests in Tab. 1. In this section, we investigate their claims one by one and conclude that they are invalid or irrelevant as the solution of the fake plateau problem. ${ }^{2}$

\subsection{Operator dependence}

Authors of Ref. [5] compared the ground state energy of the $N N$ system in ${ }^{1} S_{0}$ and ${ }^{3} S_{1}$ channels among NPL2013 [6, 7], CalLat2017 [15] and NPL2017 [17], all of which employed same gauge ensembles, and concluded that the source operator dependence is not established, since the $\chi^{2}$ test on the assumption that all results are eqaul gives $P$-values (significances) ranging from $8 \%$ to $65 \%$.

However, comparing all data at once is not an optimal way to investigate the operator dependence. Instead, it is better to perform the one-to-one comparison for a maximal detection of the dependence. Such comparisons are made for NPL2013 and CalLat2017 in Tab. 2, where operator dependence is clearly seen between the center of mass and the moving systems in ${ }^{1} S_{0}$ of NPL2013, between non-displaced and displaced source operators of CalLat2017, and between NPL2013 and CalLat2017 (displaced).

Furthermore, an absence of the operator dependence for the limited sets of operators, even if it is true, does not mean the absence of the "fake plateau problem". One has to prove separately that the plateau is not fake.

It is also claimed in Refs. [5, 15], without any numerical evidences, that the result from the displaced source operator in CalLat2017 does not corresponds to the ground state energy, so that no operator dependence exists in CalLat2017. ${ }^{3}$ This interpretation, however, brings other problems. If the plateau of the displaced operator corresponds to the excited scattering state, the negative energy shift of this state completely disagrees with the positive value from other sources with non-zero momentum. Thus it creates another huge operator dependence. If this plateau corresponds to the second

\footnotetext{
${ }^{2}$ It was very strange that the version 1 of Ref. [5] had not cited the commented paper [3] as the reference,

${ }^{3}$ The argument in Ref. [15] that the displaced operator does not couple to the ground state does not have solid ground.
} 
Table 2. $\chi^{2} /$ dof ( $P$-value $\left.\%\right)$ between data of the ground state energy using NPL2013 configurations: NPL $(0,2)$ and $\operatorname{CL}(0, d)$, where NPL $(0)$ and NPL(2) means NPL2013 [6, 7] in the center of mass and the moving systems, respectively, while CL(0) and CL(d) are CalLat2017 [15] with non-displaced and displaced source operators, respectively. The upper-right (lower-left) triangle corresponds to $L=24(32)$. As possible correlations between two data are neglected here, $\chi^{2}$ in this table might be underestimated.

\begin{tabular}{|c||c|c|c||c|c|c|c|}
\hline \multicolumn{1}{|c||}{} & \multicolumn{3}{c||}{$N N\left({ }^{1} S_{0}\right)$} & \multicolumn{4}{c|}{$N N\left({ }^{3} S_{1}\right)$} \\
\hline & NPL(0) & NPL(2) & CL(0) & NPL(0) & NPL(2) & CL(0) & CL(d) \\
\hline NPL(0) & - & $3.7(5 \%)$ & $0.3(56 \%)$ & - & $2.4(12 \%)$ & $0.4(52 \%)$ & $0.5(47 \%)$ \\
NPL(2) & $4.2(4 \%)$ & - & $2.6(11 \%)$ & $2.7(10 \%)$ & - & $1.1(31 \%)$ & $5.3(2 \%)$ \\
CL(0) & $0.3(59 \%)$ & $2.5(11 \%)$ & - & $1.5(22 \%)$ & $0.5(50 \%)$ & - & $2.4(12 \%)$ \\
CL(d) & $5.0(3 \%)$ & $17(<1 \%)$ & $8.6(<1 \%)$ & $11(<1 \%)$ & $24(<1 \%)$ & $31(<1 \%)$ & - \\
\hline
\end{tabular}

shallow bound state, on the other hand, one has to explain why NPL2013 missed this state, and the effective range expansion (ERE) in NPL2013 or CalLat2017 should be drastically modified as in Fig. 2 (Right), to satisfy the physical pole condition [3].

As we confirmed above, the source dependences exists in NPL2013/CalLat2017, which strongly suggest that their extractions of energy from plateaux suffer from the "fake plateau problem".

Refs. [5] also claimed that data at the largest volume $(L=48)$ is important to determine the binding energy in the infinite volume extrapolation. Although this is true, the large volume data has nothing to do with the validity of data at smaller volumes discussed in Ref. [3].

\subsection{Volume scaling of energies}

Showing that $N N$ energies in NPL2013 are almost volume independent, authors of Ref. [5] claimed that this volume independence rules out the possibility that the plateau structures are caused by the cancelations among excited states. This claim, however, is not valid in general, since the volume dependence of the fake plateau is not known. Indeed, while energies in YKU2012 [11] are almost volume independent, the corresponding ERE is very singular as shown in Ref. [3] and the source/sink operator dependences are observed as seen in the previous section. This means that the volume independence does not guarantee the correctness of data, contrary to the claim of Ref. [5].

\subsection{Physical pole condition}

Authors of Ref. [5] argued that EREs in NPL2013 satisfy the physical pole condition

$$
\left.\frac{d}{d k^{2}}\left[k \cot \delta_{0}(k)-\left(-\sqrt{-k^{2}}\right)\right]\right|_{k^{2}=-k_{b}^{2}}<0
$$

where $k_{b}^{2}$ correspond to the bound state pole, by showing that the slope of $-\sqrt{-k^{2}}$ at $k^{2}=-k_{b}^{2}$ is larger than the slope of ERE (effective range).

This argument, however, is insufficient to check the physical pole condition, which means that all intersection points between the ERE and the pole condition satisfy eq. (2). Fig. 3 shows $k \cot \delta_{0}(k) / m_{\pi}$ as a function of $\left(k / m_{\pi}\right)^{2}$, together with the NLO ERE line, taken directly from figures in NPL2013 and replotted in Ref. [3]. As the red line in both channels appears below the black solid line at $\left(k / m_{\pi}\right)^{2} \simeq 0$ and $\left(k / m_{\pi}\right)^{2} \simeq-0.13$, the ERE line must intersect with the pole condition twice, so that the deeper one inevitavely violates the physical pole condition. We therefore put 'No' on sanity check (iii) for $N N\left({ }^{1} S_{0}\right)$ and $N N\left({ }^{3} S_{1}\right)$ in the original assessment [3]. ${ }^{4}$

\footnotetext{
${ }^{4}$ Since the light red error band goes above the pole condition at $\left(k / m_{\pi}\right)^{2} \simeq-0.13$ for $N N\left({ }^{3} S_{1}\right)$, we will change 'No' to '?' in the revised version of Ref. [3].
} 

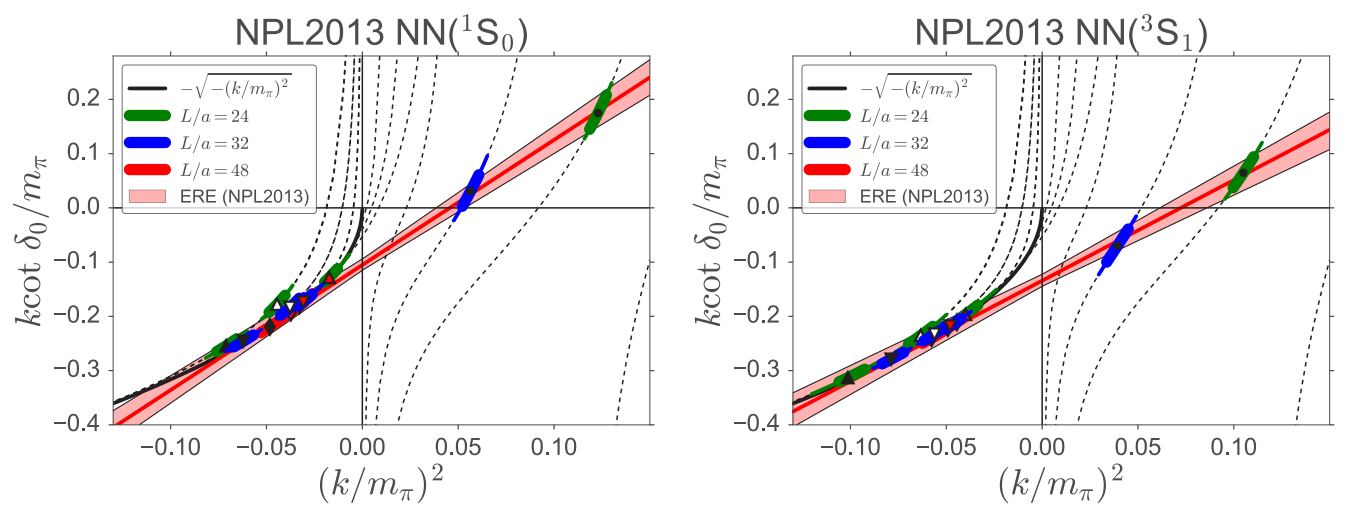

Figure 3. $k \cot \delta_{0}(k) / m_{\pi}$ as a function of $\left(k / m_{\pi}\right)^{2}$ for $N N\left({ }^{1} S_{0}\right)$ (Left) and $N N\left({ }^{3} S_{1}\right)$ for NPL2013 data. The black solid line represents the bound state condition $-\sqrt{-\left(k / m_{\pi}\right)^{2}}$, while NLO ERE fits are shown by red lines, together with light red error bands.

Ref. [5] states that "The incorrect conclusions reached in HAL potentially arise from attempting to reanalyze highly correlated data, such as the encountered in the phase-shift analysis, without making use of those correlations". ${ }^{5}$ However, this statement is not correct, since the ERE line in the figure is NOT our reanalysis but is taken from the figure in NP2013, as already mentioned.

\section{Some other concerns on Ref. [5]}

\subsection{Inadequate data handling}

In Ref. [5], authors said that they made new ERE analyses to their data and claimed that data passed the sanity check. As already shown, our sanity check in Ref. [3] was applied to EREs in NPL2013 but not to new analysis, so that the statement in Ref. [5] that " the results of Refs.[6.7](=NPL2013) pass this check, in stark contrast to the claims of HAL" is inadequate. Moreover, we found that values of the energy shift were modified from NPL2013, as summarized in Tab. 3, where data in NPL2013 are compared with data read off from figures in Ref. [5], and we pointed out at this conference that criticisms based on data modified from the original ones cannot be taken as face value. ${ }^{6}$

\subsection{Incorrect fitting}

The ERE for ${ }^{1} S_{0}$ in Refs. [5, 8] does not intersect with the line of the Lüscher formula at $L=24$, as shown in Fig. 4 (Left). Since the $\chi^{2}$ cannot be defined in this case, it is not clear how authors of Ref. [5] obtained the line and its errors in their analysis. Since the vertical error depends on the horizontal one, we have to fit lattice data by the ERE with the constraint due to the Lüscher's finite volume formula, as demonstrated in Fig. 4 (Right): Once the ERE line is fixed by given fit parameters, an intersection between the ERE and the Lüscher's formula on each $L$ is determined (black square), and then $\left(k / m_{\pi}\right)^{2}$ of the intersection is compared with that of lattice data (blue circle) to calculate the corresponding $\chi^{2}$. As the correct $\chi^{2}$ cannot be defined without intersection, it is probably that Ref. [5, 8] did not take into account this constraint correctly.

\footnotetext{
${ }^{5}$ After the lattice conference, the version 2 of [5] appeared without this sentence.

${ }^{6}$ Although this data modification is mentioned in its version 2, there remains a similar statement that "the results of Refs.[12.13](=NPL2013) pass this check, contrary to the claims in HAL".
} 
Table 3. $E_{N N}-2 m_{N}(\mathrm{MeV})$ for the ground state (upper) and the first excited state (lower) for various volumes in NPL2013 and Ref. [5], where $d$ denotes the total momentum $P=2 \pi d / L$.

\begin{tabular}{|c|c|c|c|c|c|c|c|}
\hline & & \multicolumn{3}{|c|}{$N N\left({ }^{1} S_{0}\right)$} & \multicolumn{3}{|c|}{$N N\left({ }^{3} S_{1}\right)$} \\
\hline$d$ & $\mathrm{~L}$ & 24 & 32 & 48 & 24 & 32 & 48 \\
\hline 0 & NPL2013 & $-17.8(3.3)$ & $-15.1(2.8)$ & $-13.1(5.2)$ & $-25.4(5.4)$ & $-22.5(3.5)$ & $-19.7(5.2)$ \\
\hline 0 & [5] & $-19.0\left(\begin{array}{l}2.5 \\
2.2\end{array}\right)$ & $-18.5\left(\begin{array}{l}2.4 \\
2.1\end{array}\right)$ & $-19.9\left(\begin{array}{l}3.4 \\
4.3\end{array}\right)$ & $-28.5\left(\begin{array}{l}1.7 \\
1.7\end{array}\right)$ & $-26.3\left(\begin{array}{l}2.7 \\
2.2\end{array}\right)$ & $-25.3\left(\begin{array}{l}3.9 \\
4.9\end{array}\right)$ \\
\hline 2 & NPL2013 & $-28.5(4.5)$ & $-24.9(3.8)$ & $-19.3(4.4)$ & $-40.7(8.3)$ & $-31.6(4.2)$ & $23.1(6.8)$ \\
\hline 2 & [5] & $-23.5\left(\begin{array}{l}3.3 \\
2.7\end{array}\right)$ & $-21.4\left(\begin{array}{c}2.3 \\
2.4\end{array}\right)$ & $-21.8\left(\begin{array}{c}4.2 \\
5.5\end{array}\right)$ & $-34.3(3.6)$ & $-29.6\left(\begin{array}{l}3.3 \\
2.8\end{array}\right)$ & $-27.5\left(\begin{array}{l}4.4 \\
5.4\end{array}\right)$ \\
\hline 0 & NPL2013 & $48.7(2.8)$ & $22.5(3.5)$ & & $41.6(3.8)$ & $15.7(3.8)$ & \\
\hline 0 & [5] & $49.3\left(\begin{array}{l}3.0 \\
2.4\end{array}\right)$ & $21.3(2.4)$ & & $37.5\left(\begin{array}{l}3.8 \\
3.7\end{array}\right)$ & $12.5\left(\begin{array}{l}3.2 \\
2.9\end{array}\right)$ & \\
\hline 2 & [5] & $44.0\left(\begin{array}{l}4.3 \\
3.4\end{array}\right)$ & $18.7\left(\begin{array}{l}2.4 \\
2.7\end{array}\right)$ & & $28.9\left(\begin{array}{l}5.2 \\
4.5\end{array}\right)$ & $8.4\left(\begin{array}{l}2.4 \\
2.1\end{array}\right)$ & \\
\hline
\end{tabular}
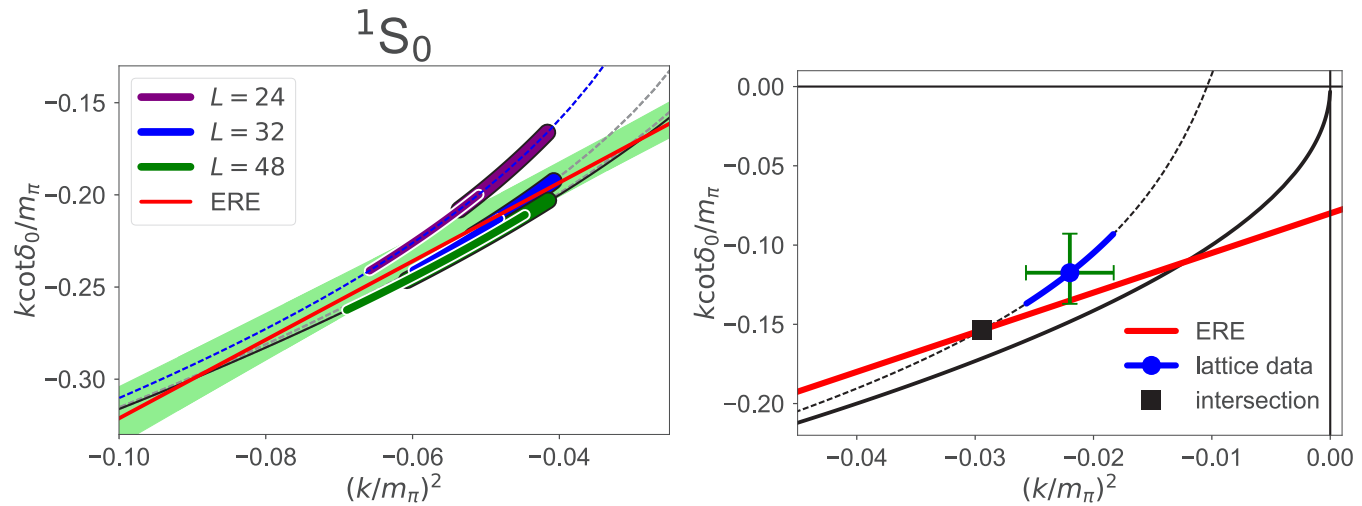

Figure 4. (Left) The ERE (red line) for ${ }^{1} S_{0}$ of Refs. $[5,8]$ and the finite volume formula (dashed lines). (Right) The correct error analysis with the constraint.

\subsection{Lattice spacing and pion mass}

NPL QCD collaboration employed the SU(3) flavor symmetric QCD configurations for various publications [5-8, 17-26]. While the lattice spacing $a$ in Refs. [5-8, 18, 20, 24] ( $a \simeq 0.15 \mathrm{fm})$ is different from $a$ in Refs. [17, 19, 21-23, 25, 26] ( $a \simeq 0.12 \mathrm{fm}$ ), the pion mass $m_{\pi}$ in these references is always the same $\left(m_{\pi} \sim 806 \mathrm{MeV}\right)$. Although the lattice spacing can depend on the physical input $\left(r_{0}\right.$, light hadron masses, heavy meson spectra, etc.), the pion mass must be changed accordingly. It is not clear how these values are obtained and how the chiral and/or continuum extrapolation are made.

\section{Summary}

As we discussed in this report, our sanity check in Tab. 1 remains valid, contray to the claims in Refs. $[4,5]$, which contain invalid statements or inadequate data handling or both.

\section{References}

[1] T. Iritani et al., JHEP 10, 101 (2016), 1607.06371 
[2] S. Aoki, T. Doi, T. Iritani (2016), [PoSLATTICE2016,109(2016)], 1610.09763

[3] T. Iritani, S. Aoki, T. Doi, T. Hatsuda, Y. Ikeda, T. Inoue, N. Ishii, H. Nemura, K. Sasaki (2017), (accepted for publication in Phys. Rev. D), 1703.07210

[4] T. Yamazaki, K.I. Ishikawa, Y. Kuramashi, A. Ukawa (PACS), PoS LATTICE2016, 108 (2017), 1702.00541

[5] S.R. Beane et al. (2017), 1705.09239

[6] S.R. Beane, E. Chang, S.D. Cohen, W. Detmold, H.W. Lin, T.C. Luu, K. Orginos, A. Parreno, M.J. Savage, A. Walker-Loud (NPLQCD), Phys. Rev. D87, 034506 (2013), 1206.5219

[7] S.R. Beane et al. (NPLQCD), Phys. Rev. C88, 024003 (2013), 1301.5790

[8] M.L. Wagman, F. Winter, E. Chang, Z. Davoudi, W. Detmold, K. Orginos, M.J. Savage, P.E. Shanahan (2017), 1706.06550

[9] M. Luscher, U. Wolff, Nucl. Phys. B339, 222 (1990)

[10] T. Yamazaki, Y. Kuramashi, A. Ukawa (PACS-CS), Phys. Rev. D84, 054506 (2011), 1105.1418

[11] T. Yamazaki, K.i. Ishikawa, Y. Kuramashi, A. Ukawa, Phys. Rev. D86, 074514 (2012), 1207.4277

[12] T. Yamazaki, K.i. Ishikawa, Y. Kuramashi, A. Ukawa, Phys. Rev. D92, 014501 (2015), 1502.04182

[13] S.R. Beane, E. Chang, W. Detmold, H.W. Lin, T.C. Luu, K. Orginos, A. Parreno, M.J. Savage, A. Torok, A. Walker-Loud (NPLQCD), Phys. Rev. D85, 054511 (2012), 1109. 2889

[14] K. Orginos, A. Parreno, M.J. Savage, S.R. Beane, E. Chang, W. Detmold, Phys. Rev. D92, 114512 (2015), 1508.07583

[15] E. Berkowitz, T. Kurth, A. Nicholson, B. Joo, E. Rinaldi, M. Strother, P.M. Vranas, A. WalkerLoud, Phys. Lett. B765, 285 (2017), 1508.00886

[16] E. Berkovitz (CalLat), Calm Multi-Baryon Operators, in Proceedings, 35th International Symposium on Lattice Field Theory (Lattice2017): Granada, Spain (2018), Vol. unknown, p. unknown, unknown

[17] B.C. Tiburzi, M.L. Wagman, F. Winter, E. Chang, Z. Davoudi, W. Detmold, K. Orginos, M.J. Savage, P.E. Shanahan (2017), 1702 . 02929

[18] S.R. Beane, S.D. Cohen, W. Detmold, H.W. Lin, M.J. Savage, Phys. Rev. D89, 074505 (2014), 1306.6939

[19] S.R. Beane, E. Chang, S. Cohen, W. Detmold, H.W. Lin, K. Orginos, A. Parreno, M.J. Savage, B.C. Tiburzi, Phys. Rev. Lett. 113, 252001 (2014), 1409. 3556

[20] S.R. Beane, E. Chang, S.D. Cohen, W. Detmold, H.W. Lin, K. Orginos, A. Parreno, M.J. Savage, Phys. Rev. D91, 114503 (2015), 1410. 7069

[21] S.R. Beane, E. Chang, W. Detmold, K. Orginos, A. Parreno, M.J. Savage, B.C. Tiburzi (NPLQCD), Phys. Rev. Lett. 115, 132001 (2015), 1505.02422

[22] E. Chang, W. Detmold, K. Orginos, A. Parreno, M.J. Savage, B.C. Tiburzi, S.R. Beane (NPLQCD), Phys. Rev. D92, 114502 (2015), 1506.05518

[23] W. Detmold, K. Orginos, A. Parreno, M.J. Savage, B.C. Tiburzi, S.R. Beane, E. Chang, Phys. Rev. Lett. 116, 112301 (2016), 1508.05884

[24] A. Parreno, M.J. Savage, B.C. Tiburzi, J. Wilhelm, E. Chang, W. Detmold, K. Orginos, Phys. Rev. D95, 114513 (2017), 1609.03985

[25] M.J. Savage, P.E. Shanahan, B.C. Tiburzi, M.L. Wagman, F. Winter, S.R. Beane, E. Chang, Z. Davoudi, W. Detmold, K. Orginos (2016), 1610.04545

[26] P.E. Shanahan, B.C. Tiburzi, M.L. Wagman, F. Winter, E. Chang, Z. Davoudi, W. Detmold, K. Orginos, M.J. Savage (2017), 1701.03456 